Freiza Niella, M., Vilà Baños, R., Rubio Hurtado, M.J. \& Belghirane El Moussaoui, F. (2017). Los futuros pedagogos ante el hecho religioso. Estudio exploratorio en la Universidad de Barcelona. Revista Electrónica Interuniversitaria de Formación del Profesorado, 20 (3), 195207. DOI: http://dx.doi.org/10.6018/reifop.20.3.282501

\title{
Los futuros pedagogos ante el hecho religioso. Estudio exploratorio en la Universidad de Barcelona
}

Montserrat Freixa Niella, Ruth Vilà Baños, M.José Rubio Hurtado, Fatima Belghirane El Moussaoui

Universidad de Barcelona

\section{Resumen}

Se presenta un estudio exploratorio sobre la diversidad religiosa-cultural y el diálogo interreligioso surgido de la investigación financiada por la Generalitat de Cataluña "Gestionar la diversitat religiosa i cultural des de l'educació. Els Futurs agents socioeducatius" (2014 RELIG 00019). Se analizan los planes docentes de los grados de la facultad de Educación de la Universidad de Barcelona y se valida un instrumento de medición de las actitudes de los futuros agentes socioeducativos aplicándolo a una muestra de estudiantes de último curso de Pedagogía. Los resultados indican actitudes moderadamente altas y la importancia de estar formado para promover el diálogo interreligioso.

\section{Palabras clave}

Diálogo interreligioso e intercultural; religión; pedagogía; educación superior

\section{The future pedagogues before the religious fact. Exploratory study at the University of Barcelona}

\begin{abstract}
We present the results of an exploratory study about the religious diversity and interfaith dialogue. This study was funded by the Generalitat of Catalonia "Gestionar la diversitat religiosa i cultural des de l'educació. Els Futurs agents socioeducatius" (2014 RELIG 00019). We analyze teaching plans of the degrees of the Faculty of Education at the University of Barcelona. We validate an instrument for measuring the attitudes of future socio agents.
\end{abstract}

\section{Contacto:}

Montserrat Freixa Niella, mfreixa@ub.edu, Departamento de Métodos de Investigación y Diagnóstico en Educación. Facultad de educación. Universidad de Barcelona, Barcelona. 08035. estudio exploratorio sobre la diversidad religiosa-cultural y el diálogo interreligioso surgido de la investigación financiada por la Generalitat de Cataluña "Gestionar la diversitat religiosa i cultural des de l'educació. Els Futurs agents socioeducatius" (2014 RELIG 00019) 
We apply it to a sample of students in their final year of Pedagogy. The results indicate moderately high attitudes and the importance of being formed to promote interfaith dialogue.

\section{Key words}

Interreligious and intercultural dialogue; religion; pedagogy; higher education

\section{Introducción}

Al acceder a la Universidad, los estudiantes de Pedagogía de la Universidad de Barcelona llevan en su mochila un bagaje sobre el hecho religioso. Durante su formación inicial, la van llenando de todo tipo de contenidos. Las actitudes de este alumnado sobre la diversidad religiosa, el diálogo intercultural e interreligioso y el papel de la educación se erigen como uno de los principales requisitos para su futura labor profesional puesto que ésta se basa en la relación educativa con el otro. Así, establecer una relación educativa con un otro que es mirado al inicio como diferente, significa también saber gestionar pedagógicamente la diversidad religiosa.

Este artículo presenta el análisis de los planes docentes de los grados de la Facultad de Educación de la Universidad de Barcelona. Seguidamente, se indican los resultados de la validación de un instrumento para analizar las actitudes de los futuros agentes socioeducativos ante la diversidad religiosa, el diálogo intercultural e interreligioso y el papel de la educación. Finalmente, muestra los resultados de la prueba piloto del instrumento en los estudiantes del último curso del grado de Pedagogía de esta Universidad.

\section{La diversidad religiosa, el diálogo interreligioso y el grado de Pedagogía.}

España tiene una larga tradición de pluralismo religioso y cultural que se vio truncada con la dictadura franquista y su imposición del catolicismo como religión única, reconociéndola como la religión oficial del Estado. Como indica Tamayo (2005), actualmente en nuestra sociedad todavía quedan vestigios de esta confesionalidad, aunque muchas voces tanto del mundo académico como del político explican que se está recuperando el pluralismo religioso. En este sentido, Estruch $(2014,5)$ apunta que la diversidad religiosa existe en nuestro contexto "no tanto por la posibilidad de escoger entre una multiplicidad de opciones, como por el hecho de poder libremente aceptar o rechazar el antiguo monopolio religioso".

En Cataluña, la diversidad religiosa no es un fenómeno de las últimas décadas puesto que hace ya más de cien años de la implantación de comunidades protestantes o adventistas y algo menos de judíos o testimonios de Jehová (Estruch, 2014). Lo que ha sucedido es que la diversidad religiosa ha dejado de ser invisible teniendo presencia en el espacio público y se ha acelerado e incrementado por los flujos migratorios de la población musulmana, de los países de la Europa del este y de América latina Y aunque la religión católica sigue siendo la mayoritaria en el Estado español, la realidad actual (Vendrell et al, 2004), indica unas tendencias hacia una secularización cada vez más acusada. Así, en España se ha pasado de un $8 \%$ en 1978 de personas que se declaran adscritas a opciones de conciencia no religiosa, al $25 \%$ en el año 2013. La realidad territorial es también diversa, siendo Catalunya la autonomía que presenta el porcentaje más elevado de conciencia no religiosa, el 40\%. 
Se debe entender la diversidad religiosa como una oportunidad previniendo los posibles factores de valores e intereses (Generalitat de Catalunya, 2013) o como una posibilidad de enriquecimiento mutuo y de convivencia renovada y profunda (Torradeflot, 2012) para llegar a una nueva sensibilidad interreligiosa. Para ello, se hace necesaria la gestión de esta diversidad religiosa mediante el diálogo interreligioso. Según Santiago y Corpas (2012), éste debe implicar una predisposición para cuestionarse las ideas propias a la luz de la de los demás. De esta forma, se abre la puerta para un enriquecimiento y transformación mutuos entre los participantes del diálogo. El diálogo implica el intercambio respetuoso y con apertura de miras de las distintas perspectivas entre los grupos y personas con legados culturales, religiosos y lingüísticos diferentes (Elósegui, 2009). La gestión de la diversidad y el diálogo interreligioso deben encuadrarse en un modelo de laicidad para dar cabida a la pluralidad religiosa a partir del reconocimiento mutuo (Elósegui, 2009; Coll 2012). Esta pluralidad se encuentra en la esfera pública, entendida como los espacios donde interactúa la sociedad civil.

Así, la diversidad religiosa se hace visible en los distintos entornos e instituciones, como el educativo. La escuela es la única institución que acoge a toda la infancia, sin exclusión. Por ello, tiene un papel primordial en la construcción de una buena convivencia entre los niños y la niñas de diversas tradiciones espirituales y religiosas. Así la escuela se convierte en un lugar privilegiado para poder desarrollar el diálogo interreligioso. De hecho, la Fundación Migra Studium (2012) indica que éste puede verse favorecido desde la educación siendo una herramienta para la prevención y mediación de conflictos (Torradeflot, 2012) al promover el respeto, la comprensión, la tolerancia y el conocimiento del otro. Pero, aunque el conocimiento del otro no garantiza el diálogo, es un requisito que si se plantea con un espíritu crítico y empáticamente, favorece esta cultura del diálogo (Moliner y Aguilar, 2010).

Distintas formaciones universitarias, especialmente todas aquellas definidas como profesiones de relación con el otro deberían ofrecer una formación sobre la diversidad religiosa, puesto que la sociedad está inmersa en el pluralismo religioso. Estos profesionales se relacionan con "otros" estableciendo una comunicación a partir de los propios códigos culturales y religiosos y de las imágenes pre-construidas que pueden ser negativas porque el "otro" es diferente y se sospecha de éste (Melloni, 2000). Según Díetz (2008) se sigue confundiendo y simplificando las diferencias étnicas, lingüísticas, nacionales y religiosas entre "nosotros" y "ellos", entre el "yo" y el "otro", contribuyendo así a fenómenos como la islamofobia. Esta construcción negativa genera desconfianza, resistencias e interferencias en la comunicación.

De entre estas titulaciones universitarias, el grado de Pedagogía debería proponer una formación para saber gestionar pedagógicamente la diversidad religiosa. Este estudiantado debería tener una actitud abierta hacia la diversidad cultural para poder ejercer su profesión sin caer en los estereotipos, prejuicios y discriminación por razón religiosa. Como personas, sus convicciones, ideologías, creencias condicionan su labor profesional (Gratacós y Ugidos, 2011). Una de las vías pedagógicas para erradicar esta discriminación recae en el afrontamiento de las percepciones de la alteridad que están profundamente arraigadas en el imaginario social. El conocimiento del otro favorece el entendimiento entre culturas y el diálogo entre religiones (Migra Studium, 2012).

Pero, ¿cómo se definen los estudiantes respecto a la religión o al hecho religioso? ¿Qué tipo de formación han recibido durante su escolaridad obligatoria? Según Vendrell et. Ali (2014), el $50 \%$ de la juventud nacida a partir de 1979, es decir que en el momento del estudio tenían entre 18 y 34 años, declaran adscribirse a opciones de conciencia no religiosa. Más de la mitad del alumnado escolar del estado español no cursa la asignatura de religión sino las actividades formativas alternativas. En los centros públicos este porcentaje es del $58 \%$ 
mientras que en los centros privados solamente un 20\%. En éstos se aprecian diferencias siendo los centros privados no concertados los que tienen un porcentaje mayor, el $33 \%$ frente al $19 \%$ de los centros privados concertados. Estas tres tipologías de centros del sistema escolar español muestran, según Dietz (2008), la tradición de transferencia de competencias educativas a la Iglesia Católica que está todavía muy arraigada.

A partir del estudio de Vendrell et ali. (2014) se puede inferir que más de la mitad del estudiantado que accede a la Universidad no tiene una formación sobre el hecho religioso. ¿Al finalizar la educación superior, habrán llenado los pedagogos/as su mochila con algún contenido sobre la diversidad religiosa y el diálogo interreligioso?

\section{Metodología}

En este artículo se presenta el instrumento de medida utilizado en la investigación. Los objetivos del estudio son comprobar por un lado si la educación superior prepara a los futuros agentes socioeducativos, para afrontar su práctica profesional educativa desde la perspectiva de la diversidad religiosa y el diálogo interreligioso; y por otro, conocer la actitud de estos agentes hacia la diversidad religiosa y el diálogo interreligioso. Los objetivos específicos del estudio se han pilotado en el grado de Pedagogía de la Universidad de Barcelona y son los siguientes:

1. Verificar el nivel de presencia de contenidos sobre la diversidad religiosa y cultural en los estudios de los futuros agentes socio-educativos de la facultad de Educación de la Universidad de Barcelona.

2. Proponer un instrumento válido y fiable para analizar las actitudes de los futuros agentes socio-educativos ante la diversidad religiosa, el diálogo intercultural e interreligioso y el papel de la educación.

3. Analizar las actitudes de los futuros pedagogos/as ante la diversidad religiosa, el diálogo intercultural e interreligioso y el papel de la educación.

Para dar respuesta al primer objetivo específico se realizó un análisis documental de los planes docentes de los grados de: Pedagogía, Educación social, Educación infantil y Educación Primaria. Se analizaron 167 planes docentes de las cuatro titulaciones teniendo en cuenta la presencia de contenidos relacionados con la diversidad cultural y/o religiosa.

Para dar respuesta al segundo y tercer objetivos específicos se hizo un diagnóstico basado en un estudio por encuesta sobre las actitudes del alumnado de Pedagogía hacia la diversidad religiosa y el diálogo interreligioso.

\section{Análisis de los planes docentes}

El análisis descriptivo inicial sobre la presencialidad de contenido o competencias relacionadas con la diversidad cultural y/o religiosa, pone en evidencia la poca importancia que se otorga a estos contenidos. De los 167 planes docentes analizados, sólo 17 tienen algún tema afín a estos contenidos (ver tabla 2). Supone una presencia del $10 \%$ en cuanto a asignaturas. Analizando la tipología de asignatura encontramos que tan solo 8 son de formación básica, 2 obligatorias y 7 optativas. El análisis por grados muestra como la presencia del $10 \%$ en general, supone un 12\% en Pedagogía, un 11\% en Educación Social y un 8\% en Educación Infantil y Educación Primaria, respectivamente. 
Tabla 2.

Presencia de contenidos sobre diversidad cultural o religiosa en los planes docentes.

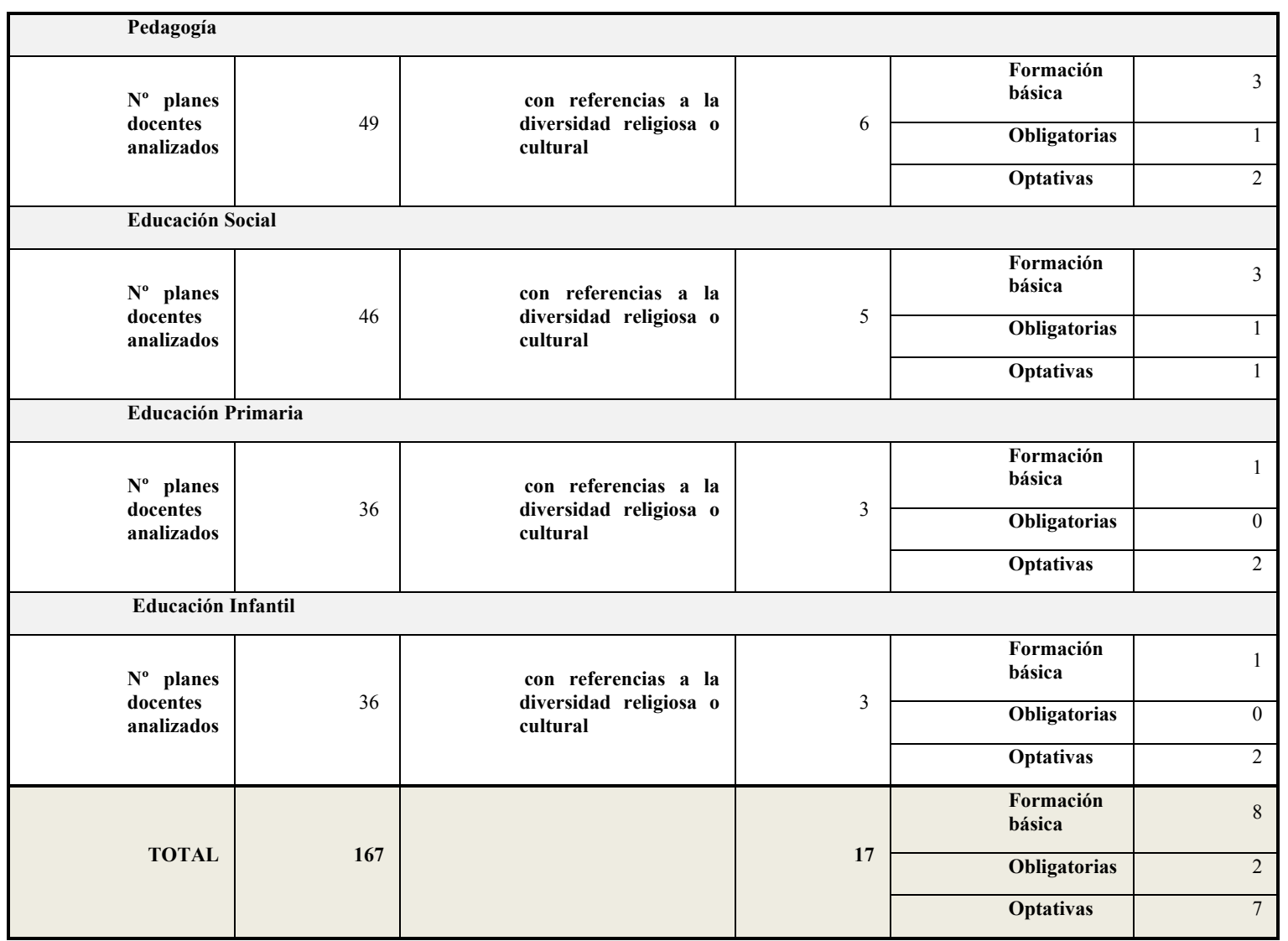

El análisis en Pedagogía muestra cómo esta presencia es todavía menor si consideramos el peso de las temáticas relacionadas con la diversidad cultural o religiosa dentro de cada plan docente identificado. Concretamente, no existe ninguna mención explícita a la diversidad religiosa ni al diálogo interreligioso. Únicamente hay referencias a la diversidad cultural en una asignatura optativa: "Interculturalidad e inmigración", destacándose los siguientes contenidos:

- Aspectos relacionados con las teorías y los análisis de los fenómenos migratorios: conceptualizaciones, terminología, visiones sociológicas, antropológicas, económicas, educativas, recorridos subjetivos, trabajo interdisciplinario.

- Aspectos relacionados con las políticas y con la gestión de la inmigración y las propuestas de interculturalidad.

En la formación básica, la asignatura "diversidad y educación" incluye la diversidad cultural conviviendo con el resto de fuentes de diversidad:

- Diversidad como conjunto de necesidades educativas especiales (NEE), recién llegados, superdotación, alumnado socialmente desfavorecido, alumnado con trastornos mentales, discapacidad física, sensorial, psíquica y motriz.

- Riesgo de considerar la diversidad como globalidad y no como conjunto de particularidades. 
Del resto de las 49 asignaturas analizadas, destacan 4 asignaturas más, que únicamente pueden llegar a intuirse temáticas afines a la diversidad cultural como son:

- “Antropología pedagógica” de formación básica, con el contenido El ser humano y el fenómeno cultural y social.

- "Pedagogía social" de formación básica con la competencia: Reconocer los elementos y los procesos en la construcción social y cultural del sujeto y las articulaciones con la educación social.

- " "Intervención educativa para la inclusión social", asignatura obligatoria que incluye las Bases para la acción en las situaciones de exclusión social, vulnerabilidad o riesgo social.

- "Gestión cultural", optativa que propone las Bases conceptuales de la gestión cultural.

\section{Estudio por encuesta}

Para conocer la actitud del alumnado de Pedagogía hacia la diversidad religiosa y el diálogo interreligioso se diseñó y validó un instrumento de medida: la "escala de actitudes ante la diversidad religiosa y cultural en la educación desde el diálogo intercultural e interreligioso". Las dimensiones e ítems propuestos en esta escala surgieron de un análisis previo de necesidades normativas (Vilà et al, 2015) y pueden verse en la tabla 1.

Tabla 1.

Especificaciones de la "escala de actitudes ante la diversidad religiosa y cultural en la educación desde el diálogo intercultural e interreligioso"

\begin{tabular}{|c|c|c|c|}
\hline DIMENSIÓN & SUBDIMENSIÓN & $\begin{array}{c}\text { Ítems } \\
\text { iniciales }\end{array}$ & $\begin{array}{l}\text { Ítems } \\
\text { finales }\end{array}$ \\
\hline \multirow{3}{*}{ Diversidad religiosa } & Factores que favorecen/dificultan la diversidad religiosa & 9 & 7 \\
\hline & Discriminación: de ciertas religiones, de la mujer, fuentes de conflicto... & 8 & 6 \\
\hline & Presencia en el espacio público & 6 & 6 \\
\hline \multirow{3}{*}{$\begin{array}{l}\text { Diálogo } \\
\text { intercultural } \\
\text { interreligioso }\end{array}$} & Concepto de religión y diálogo interreligioso & 12 & 9 \\
\hline & Elementos que favorecen el diálogo interreligioso & 7 & 6 \\
\hline & TOTAL & 19 & 15 \\
\hline $\begin{array}{l}\text { Papel de la } \\
\text { educación }\end{array}$ & TOTAL & 24 & 15 \\
\hline & TOTAL ESCALA & 66 & 49 \\
\hline
\end{tabular}

La validación de la escala se realizó en dos fases:

1) En la primera, se aplicó la estrategia del juicio de expertos para la validación de contenido mediante la cual 9 personas expertas en el ámbito de la diversidad religiosa y cultural evaluaron la escala emitiendo un juicio para cada uno de los ítems, en relación a tres aspectos: 
- Unidimensionalidad: pertinencia del ítem a la dimensión en la que se propone o pertinencia a más de una dimensión.

- Pertinencia: relación lógica del ítem con la dimensión en la que se propone.

- Importancia: importancia del ítem para los objetivos de la investigación.

En cada aspecto, la puntuación emitida fue de 1 a 3 (siendo 1 la mínima posesión de la característica y 3 la máxima).

Para cada ítem se calculó la media obtenida en el conjunto de la valoración realizada por las personas expertas (unidimensionalidad + pertinencia + importancia), eliminándose los ítems con medias iguales o inferiores a 2 y/o con desviaciones altas (que indican una gran discrepancia entre los jueces).

2) En la segunda fase se calculó la fiabilidad de la escala, una vez aplicada a una muestra de 23 estudiantes de cuarto curso de Pedagogía de la Universidad de Barcelona formada por un $91 \%$ de mujeres de 24 años de media de edad. Para el cálculo de la fiabilidad se utilizó el índice de consistencia interna Alpha de Cronbach.

La validación de contenido mediante el juicio de expertos permitió obtener una escala formada por 49 ítems, todos ellos considerados adecuados en su conjunto por los 9 jueces. Inicialmente la escala contenía 66 ítems, de los cuales 19 se eliminaron, a partir de los siguientes criterios: representatividad equitativa de ítems en las diferentes dimensiones y obtención de puntuaciones elevadas en el juicio de expertos. La tabla 3 muestra las puntuaciones medias obtenidas en cada ítem, observándose que todos superan el 2,5 de media (dentro de la media teórica de 1 a 3), una puntuación alta que indica que los ítems del instrumento son representativos del atributo que pretenden medir (la actitud hacia la diversidad religiosa y el diálogo interreligioso). Asimismo, las desviaciones bajas indican que los jueces mostraron concordancia en sus respuestas y por tanto, que están de acuerdo todos en la adecuación de la escala.

Tabla 3.

Puntuaciones medias de los ítems definitivos de la escala obtenidos en la validación de contenido mediante el juicio de expertos. ${ }^{*}$ ) (unidimensionalidad, pertinencia, importancia)

\begin{tabular}{|c|c|c|c|}
\hline & $\mathbf{N}$ & $\begin{array}{c}\text { Media } \\
(*)\end{array}$ & $\begin{array}{l}\text { Desv. } \\
\text { Tipica }\end{array}$ \\
\hline \multicolumn{4}{|l|}{ Gestión de la diversidad religiosa } \\
\hline i1: Para evitar conflictos religiosos es mejor relacionarse solamente con la propia comunidad religiosa (-) & 9 & 2,81 & 0,389 \\
\hline i2: En Cataluña existe un verdadero pluralismo religioso (-) & 9 & 2,52 & 0,671 \\
\hline $\begin{array}{l}\text { i4: El desconocimiento de las religiones que tienen las sociedades en las cuales la religión tiene poco peso fomenta la } \\
\text { incomprensión religiosa. }(+) \text {. }\end{array}$ & 9 & 2,85 & 0,293 \\
\hline i5: La diversidad religiosa puede ser una oportunidad para entender otras religiones. $(+)$ & 9 & 2,63 & 0,555 \\
\hline i6: Un Dios excluyente es fundamentalista $(+)$ & 9 & 2,52 & 0,697 \\
\hline i8: La diversidad religiosa es una amenaza para la convivencia pacífica de un territorio(-) & 9 & 2,89 & 0,258 \\
\hline 19: La diversidad religiosa es una oportunidad para perder el miedo a otras tradiciones religiosas diferentes a las mías $(+)$ & 9 & 2,85 & 0,277 \\
\hline $\begin{array}{l}\text { 111: La islamofobia se puede combatir acercando la enseñanza de la diversidad religiosa de forma transconfesional e } \\
\text { intercultural }(+)\end{array}$ & 9 & 2,74 & 0,433 \\
\hline 112: La discriminación de ciertas religiones amenaza la convivencia y la paz $(+)$. & 9 & 2,70 & 0,388 \\
\hline 113: La educación religiosa islámica sufre marginación en las escuelas públicas $(+)$. & 9 & 2,70 & 0,484 \\
\hline
\end{tabular}




\begin{tabular}{|c|c|c|c|}
\hline & $\mathbf{N}$ & \begin{tabular}{c|} 
Media \\
$(*)$
\end{tabular} & $\begin{array}{l}\text { Desv. } \\
\text { Tipica }\end{array}$ \\
\hline i14: Actualmente se ha erradicado el monopolio católico que persistía en la educación religiosa (-) & 9 & 2,66 & 0,536 \\
\hline i15: La religión musulmana es la más discriminadora con la mujer (-) & 9 & 2,85 & 0,369 \\
\hline i16: Es inadecuado que las mujeres ocupen cargos dentro de las religiones, como cura, obispo, imam, rabino (-) & 9 & 2,85 & 0,444 \\
\hline i18: Es inapropiado que el estado promueva la presencia de las religiones en el espacio público & 9 & 2,96 & 0,111 \\
\hline i19: Todas las religiones presentes en un territorio tienen derecho a ocupar el espacio público con sus lugares de culto (+) & 9 & 2,93 & 0,222 \\
\hline i20: La escuela, como espacio público, debe prohibir el uso de símbolos religiosos en alumnado y profesorado (-) & 9 & 2,70 & 0,529 \\
\hline i21 Es desagradable tener mezquitas en las ciudades (-) & 9 & 2,78 & 0,424 \\
\hline $\begin{array}{l}\text { i22: Es compatible un espacio público libre de símbolos con la aceptación de la libertad individual de llevar hiyab, cruces, } \\
\text { kipá,. }(+)\end{array}$ & 9 & 2,93 & 0,147 \\
\hline i23: El estado actúa de forma neutral cuando limita la construcción de espacios de culto que no sean la iglesias (-). & 9 & 2,96 & 0,111 \\
\hline \multicolumn{4}{|l|}{ Diálogo intercultural e interreligioso } \\
\hline $\begin{array}{l}\text { i24: Los conflictos religiosos son imposibles de resolver porque se ponen en juego las creencias y los valores innegociables. } \\
(-)\end{array}$ & 9 & 2,74 & 0,582 \\
\hline i26: El diálogo interreligioso puede contribuir a repensar conceptos y creencias arraigadas en cada uno (+) & 9 & 2,96 & 0,111 \\
\hline i27: El diálogo interreligioso puede garantizar la aceptación de las creencias de los otros. $(+)$ & 9 & 2,89 & 0,333 \\
\hline $\begin{array}{l}\text { i29: Es inmoral promover las relaciones entre los creyentes de las diferentes religiones presentes en cada barrio para } \\
\text { favorecer la cohesión social (-) }\end{array}$ & 9 & 2,89 & 0,258 \\
\hline i30: El hecho religioso forma parte de la tradición cultural $(+)$ & 5 & 2,93 & 0,149 \\
\hline i31: La religión aporta valores humanos necesarios para nuestro desarrollo como personas. $(+)$ & 5 & 2,86 & 0,298 \\
\hline $\begin{array}{l}\text { i32: Las iniciativas en materia de diálogo y diversidad religiosa deben excluir la religión musulmana por ser la más } \\
\text { intolerante (-) }\end{array}$ & 9 & 2,89 & 0,258 \\
\hline i33: La religión puede ayudar a crear un sentimiento de identidad y pertenencia a una comunidad. $(+)$ & 5 & 2,93 & 0,149 \\
\hline i35 Es necesario que el diálogo sea sensible cultural y religiosamente para llegar a soluciones pacíficas. $(+)$. & 9 & 2,93 & 0,222 \\
\hline i36: El diálogo interreligioso tiene dificultad por su falta de clarificación conceptual (+) & 9 & 2,85 & 0,369 \\
\hline i37: El fundamentalismo impide el diálogo porque se basa en intereses más allá de los religiosos $(+)$ & 9 & 2,85 & 0,277 \\
\hline $\begin{array}{l}\text { i38: El diálogo interreligioso debería centrarse tan solo en el fomento de valores compartidos por todos como la paz, la } \\
\text { solidaridad, la igualdad (-) }\end{array}$ & 9 & 2,93 & 0,222 \\
\hline $\begin{array}{l}\text { i40: Es necesario ofrecer un espacio de diálogo a las organizaciones religiosas para coordinarse entre ellas y con las } \\
\text { administraciones públicas }(+)\end{array}$ & 9 & 2,89 & 0,258 \\
\hline $\begin{array}{l}\text { i41: Es una pérdida de tiempo fomentar el diálogo con los agentes del territorio (comunidades religiosas, comerciantes, } \\
\text { usuarios de equipamiento,) (-) }\end{array}$ & 9 & 2,85 & 0,369 \\
\hline i42: Es necesario favorecer el diálogo entre las religiones para mejorar la convivencia. (-) & 9 & 2,70 & 0,618 \\
\hline \multicolumn{4}{|l|}{ Papel de la educación } \\
\hline i43: La educación debe estar al margen del pluralismo religioso presente en su contexto comunitario. (-) & 9 & 2,96 & 0,111 \\
\hline i44: La educación debe promover el diálogo entre religiones y culturas diversas (+) & 9 & 2,85 & 0,277 \\
\hline i45: La educación debe implicarse en materia de pluralismo religioso. $(+)$ & 9 & 2,89 & 0,166 \\
\hline i46: Los materiales docentes ofrecen información de rigor en materia de diversidad religiosa. (-) & 9 & 2,78 & 0,569 \\
\hline $\begin{array}{l}\text { i47: Más allá de enseñar la religión, se ha de desarrollar la sensibilidad espiritual como valor para promover el trabajo de } \\
\text { interioridad y la búsqueda de sentido(+) }\end{array}$ & 9 & 2,81 & 0,555 \\
\hline i48: Es necesario formar en espiritualidad en lugar de religión. $(+)$ & 9 & 2,78 & 0,441 \\
\hline i49: Las religiones tendrían que estar mejor consideradas dentro del currículum escolar. & 9 & 2,92 & 0,147 \\
\hline i51: Los conceptos como religión o espiritualidad deben excluirse de la educación para respetar la laicidad de la escuela..(-) & 9 & 2,85 & 0,277 \\
\hline i53: Es necesario dotar a los agentes socioeducativos de recursos e información rigurosa sobre las tradiciones religiosas. $(+)$ & 9 & 2,78 & 0,398 \\
\hline $\begin{array}{l}\text { i54: Los jóvenes solo tendrían que contar con sus familias y comunidades como fuente de información y formación } \\
\text { religiosa. (-) }\end{array}$ & 9 & 2,74 & 0,444 \\
\hline
\end{tabular}




\begin{tabular}{|c|c|c|c|}
\hline & $\mathbf{N}$ & $\begin{array}{c}\text { Media } \\
(*)\end{array}$ & $\begin{array}{l}\text { Desv. } \\
\text { Tipica }\end{array}$ \\
\hline i57: Es necesario implicar y favorecer la participación de las comunidades religiosas locales en la vida del municipio. $(+)$ & 9 & 2,67 & 0,558 \\
\hline i60: Dado que la materia de religión es optativa es necesario formar en materia religiosa al profesorado. (-) & 9 & 2,92 & 0,222 \\
\hline $\begin{array}{l}\text { 61: Es necesario promover una formación inicial y continua del profesorado para promover el diálogo interreligioso e } \\
\text { intercultural }(+)\end{array}$ & 9 & 2,74 & 0,397 \\
\hline i63: Son necesarios más cursos de especialización en religión ofrecidos por la universidad ( másteres, postgrados) $(+)$ & 9 & 2,85 & 0,369 \\
\hline $\begin{array}{l}\text { i64: Como futuro agente socio-educativo no creo que sea necesario estar formado para promover el diálogo intercultural e } \\
\text { interreligioso (-) }\end{array}$ & 9 & 2,89 & 0,258 \\
\hline
\end{tabular}

En cuanto a la fiabilidad de la escala, ésta en su totalidad considerando la escala inicial de 66 ítems, arrojó un Alpha de 0,873 , lo que indica una buena consistencia interna de sus ítems. De acuerdo con Nunnally, J.C (1978), valores mayores a 0,7 son aceptables como instrumentos de investigación. La versión final del instrumento de medida con los 49 ítems que lo componen, tiene una fiabilidad igualmente aceptable, con un alpha de Crombach de 0,828 .

Para el proceso de validación se tomó como participantes a una muestra piloto formada por 23 estudiantes de último curso del grado de Pedagogía, que presentaron las siguientes características: el 70\% manifiesta no ser creyente religioso, el 30\% indica ser creyente de diferentes confesiones del cristianismo, no obstante, el $65 \%$ manifiesta haber participado de algún ritual religioso, como por ejemplo la comunión. Aunque, en la actualidad, sólo tres alumnas participan en rituales religiosos y dos participan en las redes sociales con finalidades religiosas.

El $17 \%$ piensa que hay religiones mejor que otras y el $74 \%$ cree que algunas religiones están más cerradas al diálogo interreligioso. El 61\% tiene amistades practicantes de alguna religión diferente a la propia.

El 22\% ha tenido alguna experiencia en materia de diversidad religiosa (por ejemplo, en sus prácticas externas). El 74\% opina que es relevante estar formado como futuro pedagogo para promover el diálogo intercultural e interreligioso. El $78 \%$ cree no estar lo suficientemente formado para ello. El $87 \%$ dice no haber estudiado ningún contenido religioso durante la carrera universitaria y el $52 \%$ dice haberse formado en estas materias en otros contextos.

Las puntuaciones obtenidas en la escala por el alumnado participante son moderadamente altas. En el total y en cada una de las dimensiones de la escala las puntuaciones medias obtenidas son superiores a la media teórica posible por el instrumento (ver tabla 4).

Tabla 4.

Puntuaciones teóricas y observadas obtenidas en la escala

\begin{tabular}{|l|r|r|c|c|c|}
\hline Dimensiones & Media observada & Desviación típica & Mínima teórica & Media teórica & Máxima teórica \\
\hline Gestión de la diversidad religiosa & 86,35 & 7,68 & 23 & 69 & 115 \\
Diálogo intercultural e interreligioso & 64,09 & 6,91 & 19 & 57 & 95 \\
Papel de la educación & 85,65 & 14,49 & 24 & 72 & 120 \\
Total Escala & 236,09 & 24,80 & 66 & 198 & 330 \\
\hline
\end{tabular}


Estos resultados no ofrecen diferencias estadísticamente significativas entre creyentes y no creyentes, tampoco ante el hecho de haber estudiado o no materias de diversidad religiosa en otros espacios formativos no universitarios, ni tampoco de haber participado o no en rituales religiosos como la comunión. Así mismo, el hecho de tener o no amistades de religiones diferentes a la propia, o tener experiencia profesional en diversidad religiosa (por ejemplo, en las prácticas externas) parecen tener un efecto en la actitud hacia la diversidad religiosa y el diálogo interreligioso. Finalmente, pensar que hay religiones mejor que otras o algunas religiones más cerradas que otras al diálogo interreligioso, tampoco ofrece diferencias estadísticamente significativas entre las actitudes del alumnado.

Los aspectos que ofrecen diferencias estadísticamente significativas son la opinión sobre la relevancia de la formación en materia religiosa y la valoración de la formación recibida como suficiente o no para poder fomentar el diálogo interreligioso.

Concretamente, los estudiantes que creen que no es relevante formarse para fomentar el diálogo interreligioso tienen una actitud menos favorable hacia la diversidad religiosa y cultural en la educación desde el diálogo intercultural e interreligioso, que aquellos que creen que es relevante dicha formación (gráfico 1). Las diferencias en el total de la escala son estadísticamente significativas $(\mathrm{t}=-3.976, \mathrm{p}=0.001)$. Estas diferencias son también estadísticamente significativas respecto a la dimensión 2 , diálogo intercultural $\mathrm{e}$ interreligioso $(t=-3.383, p=0.003)$ y 3 , el papel de la educación $(t=-3.983, p=0.001)$. En cambio, estos estudiantes no tienen una actitud diferente estadísticamente significativa respecto a la dimensión 1 (diversidad religiosa).

Gráfico 1. Relevancia de la formación para el diálogo intercultural e interreligioso según la opinión del alumnado

De forma similar, aquellos estudiantes que creen no estar suficientemente formados para fomentar el diálogo interreligioso tienen una actitud más favorable que los que no identifican estas carencias formativas (gráfico 2). Estas diferencias son estadísticamente significativas en el total de la escala $(\mathrm{t}=2.751, \mathrm{p}=0.012)$, como también en la dimensión 3 referente al papel de la educación en el diálogo interreligioso $(t=2.977, p=0.007)$. 
Gráfico 2. Formación recibida sobre el diálogo intercultural e interreligioso según la opinión del alumnado

\section{Discusión y conclusiones}

El cuerpo del texto irá redactado a Candara a 11 puntos, interlineado a 1.1 y justificado, añadiendo un salto de línea una vez finalizado el epígrafe antes de comenzar el siguiente.

Una primera conclusión del trabajo es la escasa presencia de contenidos y competencias específicas en materia de diversidad cultural y religiosa en la formación de grado de los futuros agentes socioeducativos en la Universidad de Barcelona. Específicamente en el grado de Pedagogía, se pone además en evidencia la inexistencia de contenido específico sobre diversidad religiosa. Estos datos reafirman la idea que expresan los expertos sobre la necesidad de una formación en el sistema universitario sobre estas temáticas (Unesco, 2005; Torradeflot, 2011; López, 2012), tal y como ya se detectó en otro estudio (Vilà et al, 2015). Estos resultados también corroboran lo que manifiestan los estudiantes del grado de Pedagogía en la escala que presentamos en este artículo, que indican no estar lo suficientemente formados en diversidad religiosa puesto que no han estudiado ningún contenido religioso durante el grado. Al mismo tiempo se evidencia la necesidad de estar formado como futuro pedagogo/a para promover el diálogo intercultural e interreligioso según la opinión del propio alumnado. Más de la mitad además ha tenido que recurrir a otros contextos formativos para adquirir competencias en esta materia.

La realidad del sistema escolar catalán indica que estos jóvenes universitarios provienen de un contexto educativo multicultural. En este sentido, los resultados del estudio piloto muestran que el $61 \%$ tiene amistades practicantes de otra religión diferente a la propia. Pero este contacto y convivencia escolar entre los estudiantes, como indican Gratacós y Ugidos (2011) no significan unas relaciones interétnicas más igualitarias y positivas. Para cambiarlas, es necesario el "saber" pero también el "saber hacer". El 22\% de los estudiantes, durante el grado, han podido desarrollar este "saber hacer” mediante las prácticas externas.

Una segunda conclusión que se deriva de los datos es que los futuros pedagogos/as tienen un nivel actitudinal moderado respecto a la diversidad religiosa y cultural. Una parte importante del estudiantado piensa que hay religiones mejor que otras y la gran mayoría cree que algunas religiones están más cerradas al diálogo interreligioso que otras, evidenciándose con ello prejuicios y estereotipos en el alumnado. Las carencias detectadas respecto a la actitud del estudiantado participante y su formación en materia de diversidad cultural y religiosa podrían ser debidas a prejuicios fruto de la herencia histórica de nuestro país hacia la religión y las creencias religiosas (Diez de Velasco, 2012), que ha acabado, tal como señala Moreras (2006), en una laicidad ausente en este marco legal de relaciones entre el Estado español y las religiones. Los prejuicios todavía tienen una incidencia en la 
actualidad. Así, aunque el estado se define como aconfesional, sigue existiendo una fuerte tradición católica que impregna la cotidianidad de las personas, amarrada a la cultura con raíces católicas que ha estructurado la sociedad española. El ejemplo más claro de ello, lo encontramos en el sistema educativo.

En relación a estos resultados, concluimos con la importancia de la actitud ante la diversidad cultural y religiosa para valorar la necesidad de estar formado para promover el diálogo intercultural e interreligioso como futuros agentes socioeducativos. De la misma forma, dicha actitud se relaciona con la propia identificación de carencias formativas en la Universidad respecto a estas temáticas. El alumnado participante que ha manifestado tener una actitud más favorable hacia estas temáticas es el que más siente la necesidad de estar formado desde la Universidad y el que también identifica las carencias en la oferta formativa recibida. Estos datos pueden interpretarse a la luz de cómo los prejuicios por parte del estudiantado participante pueden estar influyendo en sus percepciones sobre la formación universitaria recibida y en la opinión sobre la necesidad de una formación inicial en Pedagogía sobre estas temáticas. Tal como señala Torradeflot (2012) es necesario proporcionar un marco para luchar contra los prejuicios y la discriminación en todas sus formas (la islamofobia, el antisemitismo, la cristianofobia y otras discriminaciones basadas en la religión o creencias).

Finalmente, concluimos con el aporte de un instrumento válido y fiable para medir la actitud hacia la diversidad religiosa y el diálogo interreligioso. La necesidad de conocer estas actitudes en los futuros agentes socioeducativos es más que necesaria (Weisse, 2009; Moliner y Aguilar, 2010; Vilà et al, 2015) y en todo caso, valoramos la posibilidad de ampliar este estudio a otros contextos universitarios.

\section{Referencias}

COLL, M. (2012). "Laïcitat i diversitat religiosa". Revista de la cátedra sobre diversitat social de la Universitat Pompeu Fabra. Divèrsia, 2, 3-35.

DIETZ, G. (2008). La educación religiosa en España: ¿contribución al diálogo intercultural o factor de conflicto entre religiones?, Estudios sobre las Culturas Contemporaneas, XIV (28), 11-46.

DIEZ DE VELASCO, F. (2012). Religiones en España: historia y presente. Madrid: Akal.

ELÓSEGUI, M. (2009). Políticas públicas y educación intercultural. En A J. Garay, G. Díaz, M. Elósegui y M. Sabariego. El diálogo intercultural en España: un requisito de la educación y cultura de Paz. (pp131-189) Madrid: Ministerio de Educación.

ESTRUCH, J. (2014). "La diversitat religiosa a Catalunya". Revista de la cátedra sobre diversitat social de la universitat pompeu fabra. 5, 3-12

GENERALITAT DE CATALUNYA (2011). Encuesta de preinscripción a la universidad Consultado 30 de julio de 2015 en: http://www.ub.edu/dades_academiques/estudis/preins/2011-12/P_11045.pdf

(2013). La diversitat religiosa en les societats obertes. Criteris de discerniment. Consell Assessor per a la diversitat religiosa. Barcelona. Generalitat de Catalunya, Departament de Governació i Relacions Institucionals. Barcelona. 
GRATACÓS, P. y UGIDOS, P. (2011). Diversitat cultural i exclusió escolar. Dinàmiques educatives, relacions interpersonals i actituds del professorat. Barcelona. Fundació Jaume Bofill.

LÓPEZ, M. (2012). Políticas europeas en relación con la formación en materia religiosa: la promoción del diálogo interreligioso y la educación intercultural. En J.L. Álvarez y M.A. Essomba (coords). Dioses en las aulas. Educación y diálogo interreligioso (pp6184). Barcelona: Graó.

MELLONI, X. (2000). "Els cecs i l'elefant. El diàleg interreligiòs". Quadern de Cristianisme i Justícia, 97, 1-29

MIGRASTUDIUM (2012). Memòria 2012. Creant ponts de diàleg. Barcelona, Fundació MigraStudium.

MOLINER, A. y AGUILAR, N. (2010). Les tradicions religioses en els llibres de text. Barcelona: Montflorit Edicions i Assessoraments S.L.

MORERAS, J. (2006). Migraciones y pluralismo religioso. Elementos para el debate. Barcelona: Edicions Bellaterra, S.L.

NUNNALLY, J.C (1978). Psychometric Theory. New York. McGraw-Hill.

SANTIAGO, M. y CORPAS, C. (2012). Bases para el desarrollo de buenas prácticas de educación para la convivencia en la diversidad cultural y religiosa. En J.L Álvarez. y M.A Essomba, (coords.) Dioses en las aulas. Educación y diálogo interrelgioso. (pp123141) Barcelona: Graó.

TAMAYO, J. (2005) "Razones para el diálogo interreligioso". Consultado el 12 de febrero de 2015 en Encuentros multidisciplinares, Vol. 7, № 19, 17-32.

TORRADEFLOT, F. (2011). Religiones y pluralismo. Las vías del diálogo interreligioso en España. Madrid: Observatorio del Pluralismo Religioso en España.

_ (coord) (2012). Catalunya i el diàleg interreligiós aportacions innovadores al diàleg interreligiós des dels valors de les nacions unides. Barcelona Associació UNESCO per al Diàleg Interreligiós

UNESCO (2005). The Rabat Commitment Conclusions and Recommendations of the Rabat Conference on Dialogue among Cultures and Civilizations through Concrete and Sustained Initiatives Rabat, Morocco. Consultado el catorce de mayo de 2015 en: http://www.unesco.org/dialogue/rabat/commitment.html

VENDRELL, E., PONT, J.F., CANET, E., GARCÍA-ROMERAL, G., GRIERA, M.M., MARTÍN,I., LLORENTE, I; FUNES, J; CASTELLÀ, S.J., MOLINA,V., ILLA,O., GOBERNA,R., VILLANUEVA,J., PANADERO,H., LUQUE,S., PONT,A. y FAURA,P. (2014). Informe Ferrer i Guàrdia. Aportacions per una societat laica. Barcelona. Fundació Ferrer i Guardia. Espais de Llibertat

VILÀ, R.; BURGUET, M.; ESCOFET, A. Y RUBIO, M.J. (2015). Hacia el diálogo intercultural e interreligioso: necesidades normativas para una gestión de la diversidad religiosa en el espacio público. Comunicación presentada en el V Congreso REPS: Desigualdad y democracia: Políticas públicas e innovación social. Barcelona. 5 y 6 de febrero.

WEISSE, W. (coord.) (2009). Religión en educación: Contribución al diálogo. Sugerencias del proyecto de investigación REDCO para la política. Comisión Europea. Consultado el veinticinco de enero de 2015 en: http://www.redco.uni-hamburg.de 